\title{
Funcionamiento cognitivo de niños entre 4 y 6 años para la resolución de conflictos interpersonales*
}

\section{Cognitive Functioning of Children Aged 4 to 6 in Interpersonal Conflict Resolution}

\author{
María Paola Ayala Afanador ${ }^{*}$ \\ Pontificia Universidad Javeriana, \\ Colombia \\ Jairo Andrés Montes González \\ Pontificia Universidad Javeriana, \\ Colombia
}

Recibido: 8 de agosto de 2010 Revisado: 10 de septiembre de 2010 Aceptado: 1 de noviembre de 2010

\section{Resumen}

Esta investigación se centra en el estudio de aspectos del funcionamiento cognitivo de los niños en el dominio del conocimiento social, partiendo del uso de Situaciones de Resolución de Problemas (SRP). Se busca indagar la forma en la cual los niños resuelvan conflictos interpersonales y utilicen la toma de perspectiva como habilidad social. El objetivo general del proyecto es describir los funcionamientos cognitivos de 20 niñas y niños entre cuatro y seis años de edad, para la resolución de conflictos interpersonales, a través de los desempeños obtenidos en una tarea, que simula tres escenarios con situaciones de disputa entre varios personajes. Los resultados demuestran que los niños en edad escolar son capaces de incorporar habilidades sociales desde edades tempranas. Este hecho sustenta la idea de que el desarrollo cognitivo, especialmente en el dominio social, no requiere de un funcionamiento general, sino que presenta variabilidad en periodos cortos de tiempo respecto a la resolución de conflictos interpersonales, e incluso en micro momentos dentro de la misma tarea.

Palabras clave: funcionamiento cognitivo, resolución de conflictos interpersonales, toma de perspectiva, cognición social

* $\quad$ Artículo de investigación

** Correspondencia: María Paola Ayala Afanador, Correo electrónico: michepao84@gmail.com, mpayala@javerianacali.edu.co; Jairo Andrés Montes González, jamontes@javerianacali.edu.co. Carrera de Psicología. Pontificia Universidad Javeriana, Cali, Colombia. Dirección postal: A.A. No. 26239 Cali, Colombia. 


\section{ABSTRACT}

This article focuses on children Cognitive Functioning in the social domain of knowledge. It is based on the use of problem solving tasks (PST), using a social skills perspective, to investigate the way in which children solve interpersonal conflicts. The project's overall objective is to describe the cognitive functioning of 20 children between four and sixth years of age, for interpersonal conflicts solving, through the performances obtained in a task that simulates three scenarios where there are disputes between various characters. The results show that school-age children are able to incorporate social skills from an early age and support the idea that cognitive development, especially in the social domain, does not require an overall functioning, but show variability in short periods of time compared to solving interpersonal conflicts, and even within the same task's micro moments.

Key words: cognitive functioning, interpersonal conflict resolution, perspective taking, social cognition

\section{Introducción}

Con la presente investigación se pretendió caracterizar la resolución del conflicto interpersonal en niños entre los cuatro y seis años de edad. Adicionalmente, se indagó acerca del funcionamiento cognitivo manifiesto en los menores a través de la resolución de conflictos. Estos procesos fueron abordados desde el estudio del desarrollo del conocimiento social, específicamente en lo que respecta a la toma de perspectiva.

A partir de la comprensión de un fragmento del dominio de conocimiento social, se toman en consideración los diversos conflictos a los que cotidianamente se ve enfrentado el niño y que, pese a que en ocasiones son vistos por los adultos como conflictos de mínima magnitud, exigen del niño el despliegue de toda una serie de estrategias socio-cognitivas que le ayudarán a enfrentar sus propios conflictos (Tropo-Gordon y Ascher, 2005), y a incluirse de forma activa en la sociedad. Este tipo de conflictos, se presenta en contextos educativos en los cuales el niño se interrelaciona con sus pares, siendo estas disputas elementos constitutivos de cualquier entidad educativa que incluya menores dentro de su quehacer (Moncada \& Mejía, 2003).

El conocimiento social permite concebir a los niños no sólo en relación con el mundo físico y la comprensión de éste (causalidad, planificación, clasificación, noción de número, entre otros), sino también con respecto a las relaciones sociales que establece cuando interactúa con otras personas. Dada su naturaleza social, el niño es influenciado por unas normas y lineamientos de comportamiento con respecto a los otros. A partir de aquí nace el interés de algunos autores por el desarrollo moral de los niños.

Las teorías clásicas explicativas del desarrollo moral en la primera infancia (Kohlberg, 1992, Eisenberg \& Turiel, citados por Enesco \& Navarro, 1996), coinciden con la idea de que sólo hasta que un nivel de dominio social de razonamiento o incluso del pensamiento lógico se encuentren considerablemente posicionados en el niño, éste logrará acciones morales y la inclusión de la norma en su comportamiento. Por otro lado, algunas investigaciones recientes sustentan la importancia que conlleva el conocer cómo se da en el niño aquella instauración de normas sociales, representaciones culturales y deberes que se enmarcan dentro de su desarrollo socio-cognitivo y moral. Es decir, se piensa más en un niño en positivo que no sólo aparece como producto de la interiorización de las normas sociales y éticas, sino que también se considera un sujeto que desempeñe papeles sociales y sea partícipe de su mismo desarrollo, incluso antes de alcanzar un estadio avanzado del desarrollo social y una edad considerada como típica para dar muestra de una comprensión extensa de los aspectos sociales del mundo que habita. 


\section{Método}

\section{Participantes}

Participaron diez niñas y diez niños entre cuatro y seis años de edad, pertenecientes a dos instituciones educativas privadas de la ciudad de Cali (Colombia), de un nivel socioeconómico medioalto.

\section{Diseño}

Se realizó un estudio experimental, utilizando la observación como herramienta para el registro. Los datos obtenidos permitieron describir los funcionamientos cognitivos en la toma de perspectiva (intrasujeto e intersujeto), cuando los participantes se enfrentan a tareas de resolución de problemas. Se observó el desempeño de cada niño frente a la tarea, registrado en una rejilla y posteriormente se comparó el desempeño con otros sujetos en la tarea, en tres intentos de solución. Para ello, se hizo uso de una metodología microgenética.

\section{Instrumentos}

Los niños se enfrentaron a una situación experimental (figura 1) que exige para su resolución habilidades cognitivas como la Toma de perspectiva y la Conducta prosocial. La situación de resolución del presente estudio consta de una maqueta dividida en 3 lugares o áreas (casa, colegio y parque), en las que se encuentran diferentes personajes que suscitan un tipo de situación conflictiva diferente.

Las siguientes son las categorías del estudio:

- Conducta prosocial: ésta se describe como la manera en que un sujeto desarrolla acciones que pueden beneficiar a otro (Eisenberg \& Fabes, 1998, citados por Persson, 2005).

- Toma de perspectiva: aquí se diferencia la capacidad del niño para tener en cuenta la opinión, el sentir o el pensamiento del otro personaje para la resolución del conflicto, sin necesidad de descuidar el propio criterio (Steins \& Wicklund, 1998).

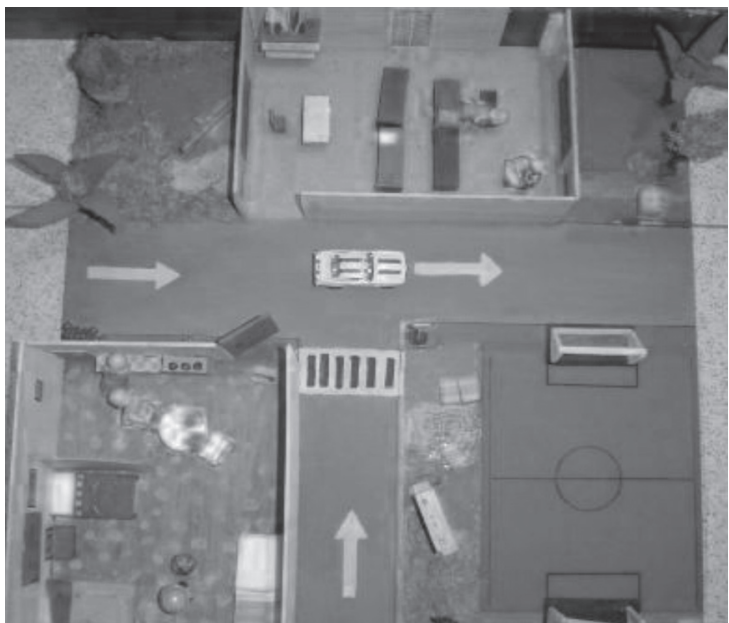

Figura 1. Situación de Resolución de problemas: tarea de resolución de conflictos.

- Evitación del conflicto: Oteros (2006) considera la evitación del conflicto como una forma en la cual el sujeto puede generar soluciones al mismo, y en la que se abandona momentáneamente la disputa, pero creando soluciones indirectas.

Resolución de la tarea: el examinador toma partido de la situación, acogiendo a uno de los personajes para representarlo y generar un tipo de conflicto interpersonal diferente en cada lugar de la maqueta. Igualmente, el examinador permite que el niño escoja el personaje a representar y le proporciona una consigna, la cual fluctúa según el momento de aplicación, tal como se describe en la tabla 1: "P1 (como sea nombrado) está en su casa, tiene que ir a la escuela a dejar una tarea y después quiere ir a jugar al parque. Debes ayudarle a $\mathrm{P} 1 \mathrm{a}$ ir al parque en pocos minutos porque sus amigos lo esperan".

En total, se combinan los tres lugares de la tarea con tres tipos de conflictos interpersonales, reconocidos como los más típicos presentes en niños de edad escolar (Dunn, Maguire \& Brown, 1995; Droisy \& Gaudron, 2003; Moncada \& Mejía, 2003; French, Pidada, Denoma, McDonald \& Lawton, 2005; Longaretti \& Wilson, 2006). De esta manera, se contrabalancea su aparición con los personajes de la tarea en sus tres aplicaciones (ver tabla 1). 
Tabla 1.

Aplicación de los tipos de conflicto, según los tres momentos de aplicación de la tarea

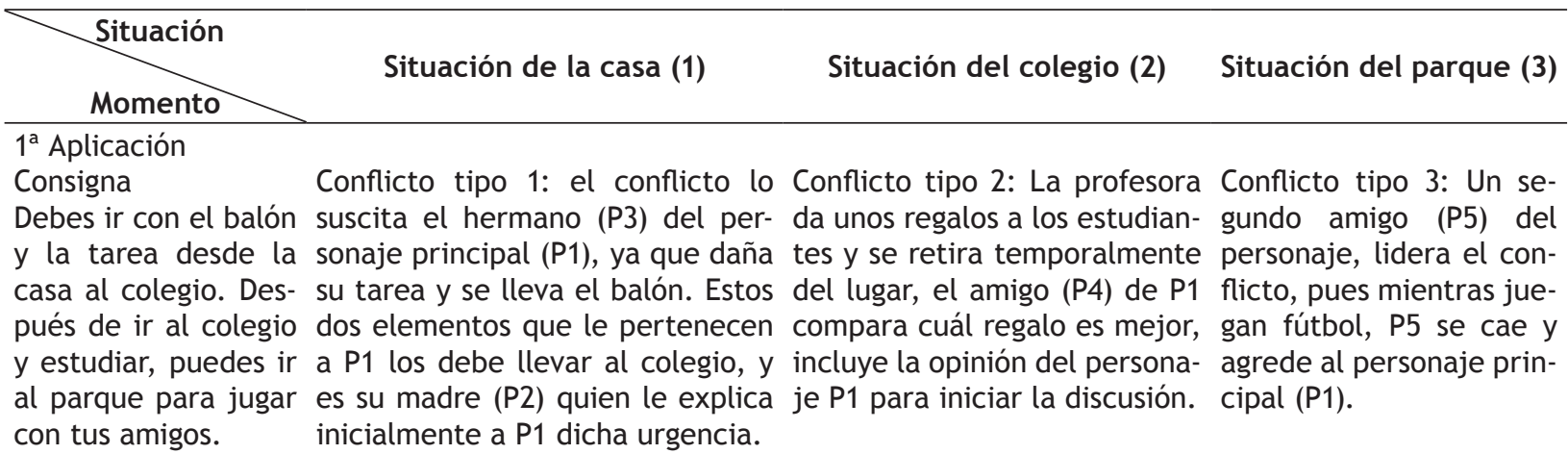

$2^{a}$ Aplicación Consigna

El niño debe levantarse temprano para ir al colegio y su hermar colegio los niños de- despierta al personaje P1. ben ir a descanso y la maestra los envía al parque después de clases.

$3^{\text {a Aplicación }}$ Consigna

Conflicto tipo 3: es un día de des- Conflicto 1: el conflicto lo ini- Conflicto tipo 2: se encanso, la madre (P2) prepara un cia el amigo del personaje P1, cuentran en el parque En la casa la madre desayuno especial para los her- pues P4 le quita la tarea que para jugar. Un amigo (P5) sirve el desayuno a manos (P1 y P3). La madre sirve ha hecho P1 y la presenta al da su opinión acerca del un solo hermano. En un desayuno primero, y el herma- docente (P2) como si fuera juego, propone otro difeel colegio, al otro no (P3) le pide el desayuno al perdía, el niño debe lle- sonaje P1 agrediéndolo. var la tarea a la profesora. Después ella los manda al parque.
Conflicto tipo 2: el personaje 1 Conflicto tipo 3: La profesora Conflicto tipo 1: mientras debe ir al colegio al otro día. La (P2) invita a los niños a pa- el personaje P1 juega madre (P2) y los hermanos ( $P 1$ sar al parque a jugar un rato con otro amigo (P5), éste P3) duermen en la casa, luego mientras ella se queda en el decide irse a su casa y su hermano (P3) se levanta en la aula. El amigo (P4) invita a P1 llevarse el balón con el a jugar en el sube y baja, el que juegan. amigo se deja caer y le reclama con golpes.
Análisis de los resultados: se tienen en cuenta las observaciones realizadas en la aplicación del instrumento y los niveles de funcionamiento cognitivo propuestos por Sastre, Moreno \& Timon (1998), en las que el sujeto actúa y se enfrenta al problema de acuerdo con modelos organizadores, seleccionando cierta información significativa del conflicto y razonando en función de ésta.
Frente a los planteamientos de los autores se construyó una rejilla de observación que diferencia tres niveles de desempeño según las acciones y verbalizaciones de los niños al resolver la tarea y se proponen dos niveles más que complementan la perspectiva de Sastre et ál. (1998). 
Tabla 2.

(Modificada) Análisis de desempeño en función de la exigencia cognitiva

\begin{tabular}{ccc}
\hline Nivel & Rasgo de desempeño (acciones) & Verbalizaciones \\
\hline
\end{tabular}

Nivel 1

En este nivel el niño puede lograr una representación del conflicto, pero no logra integrar las perspectivas de ningún personaje y prefiere evitarlo o no lo resuelve.

Subnivel $1^{a}$ (Retaliación)

En ocasiones, después de evitar la situación conflictiva o no resolver el conflicto, el niño arremete contra el personaje que originó el conflicto para beneficiarse.

\section{Nivel 2}

A partir de este nivel, los sujetos pueden representar el conflicto y lo que sucede entre los personajes. Concretan la historia y existe una movilización del sujeto por tomar la perspectiva propia, olvidando la ajena.

Nivel 3

En este nivel al igual que el anterior, el niño logra considerar el conflicto y toma partido para generar una solución. Sin embargo, aquí persiste la necesidad de tomar la perspectiva del otro (beneficiándolo), sin incluir la propia.

Nivel 4

El sujeto tiende a buscar ayuda de un tercero, personificándolo o no, para encontrar salida al conflicto interpersonal. Sin embargo, al buscar esta ayuda externa, en algunas ocasiones se prioriza la perspectiva de uno u otro personaje en el conflicto.
El niño identifica que existe un conflicto entre los personajes y las razones por las cuales se originó; sin embargo, comienza a jugar de forma indeterminada con los personajes sin concretar o proseguir el conflicto y su resolución. Incluso en ocasiones lo evita, esconde al personaje que manipula o juega con otro elemento como distractor.

El niño sabe qué sucede entre los personajes, pero resuelve el conflicto sólo beneficiándose a sí mismo y percibiendo su propio punto de vista para resolverlo.

Comprende la resolución del conflicto, pero satisface sólo sus propios intereses.

Cuando se genera el conflicto, que generalmente lo lidera el otro personaje, el sujeto considera ante todo la perspectiva del otro personaje, sin beneficiarse a sí mismo. Es decir, sin tener en cuenta su propia perspectiva para orientar una solución al conflicto.

El sujeto logra diferenciar el conflicto, evalúa las posibilidades, pero tiende a buscar ayuda de un tercero para generar soluciones. Dicha ayuda puede ser de un adulto (madre o profesor) o de un par, con la finalidad de que éste sirva de mediador y se logre dar fin al conflicto.
Cambia de tema o de situación drásticamente: ¿Ya podemos salir a jugar? ¿Y esta cosa para qué es? (haciendo alusión a algún material de la maqueta).

Esconde a su personaje del otro con quien tiene conflictos.

¡A que no me coges! ¡Estoy escondido!

¡Es mía nada más! ¡No te la voy a prestar!

Responde Sí a todo cuando el otro personaje que genera el conflicto desea la opinión del sujeto sobre cómo se solucionará.

Bueno, cógela (pelota) ¡Es tuya!

¡Profe (o mamá), mire que mi hermanito me dañó la tarea y se llevó mi pelota!

Personificando al hermano del sujeto: “¡Mi hermanito no te quiere prestar la pelota!”.

"Mamá, mire que mi hermanito me pegó porque..." 
Nivel 5

En este nivel se comprometen las perspectivas tanto del personaje que genera el conflicto, como la propia perspectiva.

El conflicto se soluciona cuando el sujeto se beneficia a sí mismo (su personaje) y al otro personaje (experimentador).
Aquí el niño logra diferenciar el conflicto entre los personajes y lo que uno y otro piensa al respecto. Así, también, logra tomar en cuenta la perspectiva del personaje que él interpreta y seguidamente la del otro personaje (examinador), para resolver el conflicto y darle fin.
Reclama a su hermanito la pelota: "Dame la pelota. Mira que no te la presté y es mi tarea que tengo que llevar al colegio".

"Juguemos otra cosa entonces, sin la pelota".

\section{Resultados}

Los resultados muestran la presencia de variabilidad cognitiva en el funcionamiento de los niños, de acuerdo con los niveles de desempeño que obtuvieron al resolver conflictos interpersonales en cada una de las tres aplicaciones de la tarea, y que a su vez equivalen a aplicaciones en periodos cortos de tiempo. Por ejemplo, en la primera aplicación se obtuvo que sus respuestas al conflicto provinieran más de la necesidad de obtener beneficio propio que beneficiar a otro o tener en cuenta su perspectiva (nivel de desempeño 2 ).

Sin embargo, hay picos altos (presencia de niveles cuatro y cinco), que demuestran que este desempeño no se da siempre de la misma manera ni permanece estable por mucho tiempo. Incluso en una misma situación los niveles varían de tal forma que no siempre un sujeto logra desempeñarse igual dentro de un mismo ensayo. Así mismo, la situación en la que más se presentan niveles altos de desempeño (puntuaciones entre 3 y 5 ) es cuando el conflicto se desarrolla en el colegio (conflicto por deseos opuestos).

En la segunda aplicación, se observó que los niños oscilan con mayor frecuencia entre los niveles de desempeño dos y uno, como en la primera aplicación de la tarea, aunque aquí se distingue mucho más la frecuencia del nivel dos. Aquí la situación conflictiva solucionada de manera más eficaz es la generada en casa y corresponde a un conflicto sobre ideas o deseos opuestos. En términos generales, es evidente que casi 17 niñas y niños del total de la muestra, logran desempeñarse en ni- veles altos (> 3), en algunas situaciones de esta aplicación.

Nivel General

\section{Desempeño General por Aplicación}

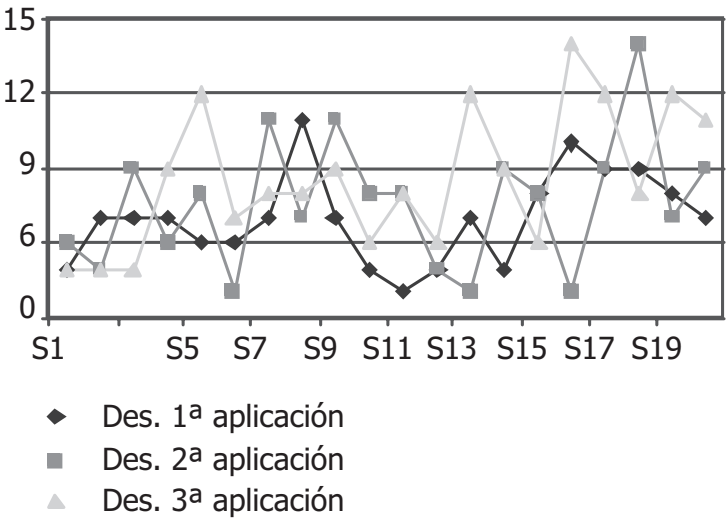

Figura 2. Desempeño general en las tres aplicaciones de la tarea. (Análisis ínter sujeto).

En la tercera aplicación se observa casi igualmente, presencia de niveles de desempeño altos, en comparación con las anteriores aplicaciones, por lo que se puede considerar que no existe una diferencia significativa entre las tres aplicaciones de la tarea. Por ejemplo, en la aplicación 1 los sujetos obtuvieron casi la misma cantidad de desempeños altos (23) que en la tercera aplicación (26), y en esta última se obtuvieron más niveles altos que en la segunda (21).

Por otro lado, se puede notar cómo los sujetos presentaron niveles altos de desempeño cuando las situaciones conflictivas se suscitaban en el parque, y cuando los conflictos se relacionaban con ideas opuestas. Así mismo, el nivel de desempeño más 
frecuente para ambos géneros en esta aplicación es el nivel dos. Sin embargo, es interesante ver cómo el nivel cinco se observa casi tan frecuente como el nivel dos en los niveles de desempeño de 10 sujetos pertenecientes a la muestra.

En los resultados se distingue igualmente los niveles de funcionamiento cognitivo de los niños y niñas, a partir de dos variables de la tarea que se han asociado a dicho desempeño: el tipo de conflicto suscitado en cada una de las situaciones y el lugar de la maqueta en el que se desarrolló la disputa. De acuerdo con el tipo de conflicto suscitado en la tarea se obtuvo que los menores logran desempeñarse en niveles altos cuando el conflicto es de tipo "ideas o intereses opuestos", es decir, cuando uno de los personajes se opone a un deseo o acción que quiere ejecutar el personaje principal. Este tipo de conflictos, generados por diferencias de intereses o ideas, fueron resueltos de manera más eficaz cuando se presentaron en la casa o en el parque, sin presencia de adultos cercanos a la situación.

Bajo circunstancias en las cuales se enfrentaban a conflictos originados por deseos opuestos u obtención de objetos, los sujetos tendieron a resolver los conflictos de manera más eficaz que cuando lo hacían frente a conflictos sobre agresiones físicas o verbales. Con lo anterior, se puede decir que el tipo de conflicto en el que mejor se desempeñaron los sujetos es el relacionado con ideasideseos opuestos.

Por otro lado, en los resultados se presenta una comparación intersujeto, en la cual se hace alusión al nivel de desempeño general de los niños en la resolución de la tarea. Aquí se consolidan los desempeños generales de los sujetos en cada aplicación de la SRP, lo que se efectuó mediante la sumatoria de las puntuaciones obtenidas en los tres momentos de la tarea y por consecuencia, en cada una de las tres aplicaciones de la misma. De acuerdo con lo anterior, se hizo posible la descripción de la variación para dicho desempeño general en las tres aplicaciones que se realizaron de la tarea. De esta manera se estableció que el nivel general de desempeño en las tres aplicaciones de la tarea que más se presenta es el ni- vel medio (entre 6 y 8). Estos desempeños fueron obtenidos a su vez a partir de la combinación de los niveles de desempeño en cada momento de la tarea. El siguiente nivel de desempeño general que más se presenta es el nivel bajo (entre 3 y 5 ), seguido por el alto (entre 9 y 12), que presenta una frecuencia similar a la del nivel bajo.

De la misma forma, se puede observar cómo los niveles de desempeño general en los sujetos, varían de acuerdo con las tres aplicaciones de la tarea sin ser esta variación necesariamente progresiva. Es decir, sin pasar de un nivel menor de desempeño a otro mayor, según se aplicaba la SRP sobre resolución de conflictos.

Así mismo, se encontraron resultados de orden cualitativo que sugieren que los menores pueden llevar a cabo diferentes maneras para resolver un conflicto, es decir, cambiar de estrategia varias veces dentro de una misma situación. Los micromomentos, se refieren a las diferentes formas en las cuales el niño acude a resolver un solo conflicto/momento en la tarea o logra generar más de una solución al conflicto suscitado. Se encontró entonces presencia de variabilidad dentro de un mismo ensayo en 19 de los 20 sujetos pertenecientes a la muestra.

Igualmente, en los resultados se describieron los perfiles de funcionamiento cognitivo de la muestra, con relación a la resolución de conflictos y a la toma de perspectiva. En este apartado se definieron cuatro perfiles clave que demuestran las diferentes formas de funcionamiento cognitivo a través del tiempo (durante las tres aplicaciones de la tarea), y la variabilidad que subyace a éstos. Se encontró que la totalidad de los sujetos tienden a funcionar cognitivamente y respecto a las tres aplicaciones de la tarea de una forma variable, es decir, con presencia de niveles altos, medios y bajos en sus desempeños. Por tal motivo, los sujetos se pudieron clasificar o agrupar según el perfil que presentaban, respecto a sus desempeños individuales relativos a cada momento de la tarea y a las tres aplicaciones de la misma.

Finalmente, se presentan los resultados correspondientes a las posibles diferencias observadas 
entre hombres y mujeres, que aunque no estaban inscritas como objetivos iniciales del presente proyecto, se tienen en cuenta como resultados del desempeño adicional. Respecto a dichos resultados, se obtuvo que en la segunda aplicación las mujeres obtuvieran más niveles de desempeño altos (> 3), que en la primera aplicación; contrario a los resultados de los hombres, quienes obtuvieron una frecuencia similar a la obtenida en la primera aplicación. Con ello, se pudo notar que en los resultados del grupo femenino se presentaron niveles mayores progresivamente en el desempeño, a diferencia de los hombres, quienes obtuvieron niveles altos de desempeño similares en las tres aplicaciones de la tarea.

Realizando una comparación de los niveles de desempeño general de los hombres se puede notar que existe cierta cercanía entre los resultados de la mayoría de los sujetos respecto a su desempeño en las tres aplicaciones a través del tiempo. Sin embargo, existen algunos niños que lograron desempeños muy diferentes con relación a las tres aplicaciones. Contrariamente, los resultados de los niveles de desempeño general muestran mucha más variación respecto a la aplicación de la tarea en diferentes momentos o tiempos. Así, también, se puede notar que los desempeños generales "Muy altos" se destacan más en las mujeres, que en los hombres.

\section{Discusión}

Al hablar de conocimiento social en el funcionamiento cognitivo se debe recordar que para que éste sea instaurado en el sujeto, de acuerdo con Flavell (2000), debe considerarse antes una serie de requisitos tales como: la necesidad que tiene el sujeto de reconocer su propia existencia y la de los otros (mundo externo), la "disposición" para realizar actos de conocimiento social a partir de lo que él conoce sobre el mundo que lo rodea y con la que se logra trascender la representación mental, $y$, finalmente, la capacidad para "inferir" los datos que proporciona el medio.

Este proceso de reconocimiento propio y externo se puede ver justificado en la SRP que se aplicó a un grupo de veinte niños de diversas edades, ya que se intentó corroborar cómo ante la necesidad de responder a una situación social hipotética sobre conflictos interpersonales, la totalidad de los niños lograron percibir no sólo la perspectiva propia y representar mentalmente el conflicto suscitado en la tarea, sino también orientar sus acciones de diferentes maneras frente a la situación social hipotética.

De acuerdo con los resultados obtenidos y acorde con algunos estudios revisados en el marco conceptual, no se requiere la previa instauración de habilidades cognitivas en el niño de manera específica, para poder considerar en él un desarrollo socio-cognitivo y de las habilidades sociales (López, Apodaca, Etxebarria, Fuentes \& Ortiz, 1998; Abello \& Amar, 2006 y Tropo-Gordon \& Ascherm, 2005, que citan a: Renshaw \& Asher, 1983; Slaby \& Guerra,1988; Murphy \& Eisenberg, 2002; Ross \& Asher, 2004; Díaz, 2005).

Con respecto a los niveles de desempeño alcanzados por los niños, no se encontraron diferencias significativas de acuerdo con el análisis intersujeto. Cabe recordar que la muestra no fue muy significativa y que los sujetos no fueron distribuidos según la variable edad, debido a que el rango de edades no era extenso y dentro de los objetivos del proyecto no se tomó en cuenta dicha distinción. Sin embargo, es importante anotar que los sujetos pertenecientes al presente proyecto no requirieron de ninguna distinción generacional para resolver la tarea propuesta.

Finalmente, en los resultados del presente estudio se reconoce un sujeto capaz de desarrollar sus habilidades sociales sin necesidad de cumplir ciertos requerimientos, como la edad, e incluso se puede cuestionar la idea de que el sujeto se desarrolla conforme cumple requisitos para escalar de un nivel a otro.

Por otra parte, se observó mediante la implementación de la Situación de Resolución de Problemas (SRP), que todos los niños lograron generar algún tipo de alternativa al conflicto y distinguían muy bien las características e intereses de los otros personajes, así como el origen y curso de la si- 
tuación conflictiva. Esto demuestra que la tarea cumple con el objetivo de elicitar (cognitivamente) las representaciones de los menores acerca del conflicto interpersonal, y puede llegar a ser una estrategia muy asertiva a la hora de describir e intervenir educativamente en el tema de la resolución de conflictos. Para Thornton (1998), citado por Henao \& Valdivieso (2006), las situaciones de resolución de problemas deben permitir que el sujeto perciba las metas de la misma, lo cual se considera como un logro del presente proyecto, ya que en términos generales los niños lograron resolver los diferentes conflictos de modo que reconocían las metas de la tarea.

La situación de resolución de problemas permitió evidenciar no sólo los discursos implicados en la representación que tienen los niños sobre los conflictos interpersonales y su resolución, sino también las acciones que finalmente desempeñan y gestos que las acompañan. Los resultados contrastan con la manera en la que algunos estudios han dado uso a metodologías, tales como: entrevistas semiestructuradas (López, et. ál., 1998; Tezer \& Demir, 2001; Arth-Pendley \& Cummings, 2002; Murphy \& Eisenberg, 2002; Droisy \& Gaudron, 2003; Moncada \& Mejía, 2003; Honeycutt, 2004, entre otros), dilemas morales (Kohlberg, Selman y Gilligan, citados por Sastre \& Moreno, 2002) o complementados con observaciones en un solo momento (Vernberg, Ewell, Berry \& Abwender, 1994; López et ál., 1998; Tezer \& Demir, 2001; Arth-Pendley \& Cummings, 2002; Murphy \& Eisenberg, 2002; Moncada \& Mejía, 2003; Droisy \& Gaudron, 2003; Honeycutt, 2004; Ross, Siddiqui, Ram \& Ward, 2004; Díaz, 2005; French et ál., 2005; Abello \& Amar, 2006; Longaretti \& Wilson, 2006; Ross, Stein \& Trabasso, 2006 y Tropo-Gordon \& Ascher, 2005), que no son suficientes para reconocer las variables implicadas en un proceso complejo como el desarrollo de las habilidades del conocimiento social.

De acuerdo con los resultados concernientes a variabilidad cognitiva, tanto en las tres aplicaciones como en los diferentes momentos de cada una de las aplicaciones de la tarea, se puede concluir que se denota cierta variabilidad en el desarrollo o funcionamiento socio cognitivo de los niños. Es así como de acuerdo con posturas más recientes (Dunn et ál., 1995; Puche, Colinvaux \& Dibar, 2001; Abello \& Amar, 2006 y Thornberg, 2006), el desarrollo de las habilidades cognitivas y sociocognitivas en el niño se aparta de los modelos referidos a cambios generales en la estructura mental, cuando el sujeto transita de un conocimiento a otro, y los sustituye por descripciones que tienen en cuenta las variaciones y especificidades en dicho desarrollo, de tal manera que permiten incluso determinar cambios dentro del mismo cambio (micromomentos). Así mismo, es posible que la variabilidad presente en este estudio, influenciada por diferentes factores, se asemeje al cambio en las estrategias de los menores cuando éstos se enfrentan a situaciones sociales reales en las que deben cambiar los objetivos y construir metas nuevas como respuesta al entorno social que suministra nueva información (Thornberg, 2006).

Siegler (2007) demuestra que la variabilidad cognitiva no implica un avance progresivo en las estrategias o herramientas cognitivas que utiliza el sujeto para alcanzar un objetivo (obtener conocimiento). Los resultados del presente estudio confirman que ante la presentación de la tarea varias veces, el niño no necesariamente logra alcanzar niveles de desempeño cada vez más altos, sino que dentro de su desempeño general se encuentran variantes y cambios en la estrategia para resolver los conflictos, que oscilan entre la toma de perspectiva de ambos personajes hasta la evitación del conflicto interpersonal. Se parte de la importancia de reconocer la ejecución de variadas estrategias (referentes a la toma de perspectiva), que el niño ha incorporado en su funcionamiento socio-cognitivo para generar solución a los conflictos, observando que existe una tendencia entre los sujetos del presente estudio a generar varios intentos de naturaleza social, antes de orientar una respuesta definitiva al conflicto hipotético que se presenta.

Respecto a la influencia del tipo de conflicto en la manera de resolverlo, se encontró que los niveles altos de desempeño se presentan generalmente cuando la disputa entre los personajes se relaciona con obtención de objetos o ideas opuestas. Es decir, que en las tres aplicaciones que se rea- 
lizaron, los menores lograron resolver conflictos de manera más prosocial, al ser dichas disputas ocasionadas por obtención de objetos y deseos opuestos (entre los personajes), a diferencia de los conflictos relacionados con agresiones físicas o verbales, pues sus niveles de desempeño oscilan entre niveles bajos. Lo anterior coincide con algunos estudios recientes (Dunn et ál., 1995; Droisy \& Gaudron, 2003; Moncada \& Mejía, 2003; French et ál., 2005; Longaretti \& Wilson, 2006 y Thornberg, 2006), que defienden la idea de que el tipo de conflicto entre niños de edad escolar puede suponer una influencia en la respuesta frente a éste.

En esta misma línea, Eisenberg \& Garvey (1981) y Ross \& Conant (1992), citados por Thornberg (2006), muestran en su estudio que los niños en edad escolar no orientan sus respuestas frente al conflicto de forma aleatoria, sino que dichas estrategias se relacionan con la manera en la que el par plantea las suyas como oponente y su rol insistente.

Con respecto al lugar o situación conflictiva, se observó en los resultados, que en algunos niños hay una tendencia a resolver conflictos conjuntamente con los adultos o a esperar la perspectiva de otro (generalmente adulto) para generar una posible solución a la disputa, siendo incluso este otro la misma experimentadora. Sin embargo, es interesante ver cómo este nivel de desempeño se presenta en relación con el lugar/situación de la maqueta en la cual se suscita el conflicto. Por ejemplo, se puede notar cómo en el lugar o situación de la casa los niños tienden a acudir a su madre $o$ adulto responsable, mientras que cuando están en el parque o en el colegio es menos frecuente acudir a estas figuras de autoridad, así estén presentes o cercanas al lugar.

Se entiende que aunque la presencia del adulto en los lugares de la maqueta, no compromete la necesidad de acudir a éste, en ocasiones los niños optaron por resolver los conflictos autónomamente cuando el adulto estaba cercano a la situación. Se puede notar que en algunos lugares en los que existen niveles altos de desempeño no se encontraban personajes adultos o terceros tan cercanos a la situación conflictiva.
Por último, se observaron algunas diferencias respecto al funcionamiento cognitivo de acuerdo con los resultados ínter género del presente estudio, sin ser éste un objetivo específico ni una muestra significativa. En lo referente a la primera aplicación, los resultados demuestran que en los hombres la frecuencia de puntajes altos en las respuestas al conflicto es mayor en comparación con las mujeres. Sin embargo, la presencia de niveles más altos en la segunda y tercera aplicación de la tarea se nota mucho más en las mujeres que en los hombres, pues en estos últimos la frecuencia de niveles altos de desempeño no varió significativamente respecto al manejo del conflicto, tal como han encontrado diferentes autores en lo concerniente a las estrategias positivas que las niñas aportan a la resolución del conflicto (López et ál., 1998; Murphy \& Eisenberg, 2002; TroppoGordon \& Ascher, 2005; Borbely, Graber, Nichols, Brooks-Gunn \& Botvin, 2005).

El cambio se presenta entonces en virtud de la recursividad del pensamiento del niño que le permite generar nuevas hipótesis, ponerlas a prueba, inferir de manera distinta y más enriquecida y de flexibilizar cada vez más su pensamiento, permitiéndose integrar información nueva y útil a sus representaciones, gracias a la articulación de sus procesos de autorregulación, planificación, monitoreo-control.

El cambio en las representaciones, no remiten a un orden secuencial en la comprensión de la situación problema, sino a un proceso dinámico y cambiante. Al interior de los intentos de resolución se muestran movilizaciones entre las puntuaciones. Estos cambios no se presentan en un movimiento progresivo, sino que durante los intentos y al realizar una mirada microgenética, el funcionamiento de los niños sufre movilizaciones entre los puntajes de las diferentes fases, mostrando reorganizaciones sucesivas que al final permiten el esbozo de representaciones más enriquecidas.

\section{Referencias}

Abello, R. \& Amar, J. (2006). Toma de perspectiva en niños pobres en Colombia. Centro de Investigaciones en Desarrollo Humano (CID- 
HUM), Universidad del Norte. Revista infancia, adolescencia y familia, 1(1), 189-202.

Arth-Pendley, G. \& Cummings, E. M. (2002). Children's Emotional Reactivity to Interadult non Verbal Conflict Expressions. Journal of $\mathrm{Ge}$ netic Psychology, l. 163(1), 97-112. Recuperado de Internet el día 5 de febrero de 2008 en: http://web.ebscohost.com/ehost/results?vi $d=8$ \&hid=114\&sid=fa6bcca9-5022-4b39-b93526d78b168cdb\%40sessionmgr102.

Borbely, C.J., Graber, J. A., Nichols, T., BrooksGunn, J. \& Botvin, G.J. (2005). Sixth Graders' Conflict Resolution in Role Playswith a Peer, Parent, and Teacher. Journal of Youth and Adolescence, 34(4), 279-291.

Díaz, M. R. (2005), La resolución de conflictos en el aula como parte del proceso de autonomía. Revista Papeles de trabajo sobre cultura, educación y desarrollo humano, 1(2), 1-22. Universidad de Castilla-la Mancha, Recuperado el 12 de febrero de 2007 desde: www.uam.es/otros/ptcedh/2005v1pdf/ v1n2esp.pdf.

Droisy, R. H. \& Gaudron, Z. Ch. (2003). Interpersonal Conflicts Resolution Strategies in Childrens: A Father-child Co-construction. Europhean Journal of Psychology of Education, 18(2), 157-169. Recuperado el 5 de marzo de 2007 desde la base de datos EBSCOHost Academic Search Elite.

Dunn, J., Maguire, M. \& Brown, J. (1995). The Development of Children's Moral Sensibility: Individual Differences and Emotion Understanding. Developmental Psychology, 31(4), 649-659.

Enesco, I. \& Navarro, A. (1996). El conocimiento social. Cuadernos de Pedagogía, 244, 69-75.

Flavell, J. (2000). El conocimiento social. En J. Flavell. (2000). El desarrollo cognitivo (pp. 13-71). España: Visor.

French, D. C., Pidada, S., Denoma, J., McDonald, K. \& Lawton, A. (2005). Reported Peer Conflicts of Children in the United States and Indonesia. Social Development, 14(3), 458-472.
Henao, B.E. \& Valdivieso, R. (2006). Coordinación de perspectivas en escolares: un estudio basado en resolución de problemas sociales cotidianos. Tesis de pregrado no publicada, Facultad de Psicología, Pontificia Universidad Javeriana, Cali.

Honeycutt, J. (2003-2004). Imagined Interaction Conflict-Linkage Theory: Explaining the Persistence and Resolution of Interpersonal Conflict in Everyday Life". Journal "Imagination, Cognition and Personality”, 23 (1), 3-26.

Longaretti, L. \& Wilson, J. (2006). The Impact of Perceptions on Conflict Management. Educational Research Quayterly, 29(4), 3-15. Recuperado el día 18 de diciembre de 2006 desde la base de datos EBSCOHost Academic Search Elite.

López, F., Apodaca, P., Etxebarria, I., Fuentes, M.J., \& Ortiz, M.J. (1998). Conducta Prosocial en Preescolares. Infancia y aprendizaje, 21(2), 45-61. Recuperado el 21 de octubre de 2006 desde: http://www.ingentaconnect. com/fias/iya/1998/00000021/00000002/ art00003

Moncada, C. \& Mejía, C.A. (2003). Representación y manejo de conflictos en niños preescolares. Revista Científica Guillermo de Ockham. 6(2), 131-143. Universidad de San Buenaventura, Cali,

Murphy, B. \& Eisenberg, N. (2002). An Integrative Examination of Peer Conflicts: Children's Reported Goals, Emotions and Behaviors. Social Development, 11(4), 534-557.

Oteros, A. M. (2006). Pautas de actuación para la resolución de conflictos en el aula. Revista Digital Investigación y Educación, 3(26), 1-11. Recuperado el día 26 de marzo de 2007 desde: http://www.csi-csif.net/andalucia/ modules/mod_sevilla/-archivos/revistaense/ n26/26080186.pdf

Persson, G. (2005). Developmental Perspectives on Prosocial and Aggressive Motives in Preschoolers' Peer Interactions. International Journal of Behavioral Development, 29(1), 80-91. 
Puche, N. R., Colinvaux, D. \& Dibar, U. (2001). El niño que piensa: un modelo de formación de maestros. Colombia: Ministerio de Educación Nacional.

Ross, H.S., Stein, N. \& Trabasso, T. (2006). How Siblings Resolve Their Conflicts: The Importance of First Offers, Planning, and Limited Opposition. Child Development, 77(6), 1730-1745.

Ross, H.S., Siddiqui, A., Ram, A. \& Ward, L. (2004). Perspectives on Self and Other in Children's Representations of Sibling Conflict. International Journal of Behavioral Development The International Society for the Study of Behavioural Development, 28(1), 37-47.

Sastre, G. \& Moreno, M. (2002). Resolución de conflictos y aprendizaje emocional. España: Gedisa.

Sastre, G., Moreno, M. \& Timón, M. (1998). Razonamiento moral y educación. Revista Educar, 22-23, 155-170.

Siegler, R. (2007). Cognitive Variability. Developmental Science, [Dev Sci] Jan, 10 (1), pp. 1049. Recuperado el día 20 de marzo de 2008 desde: http://web.ebscohost.com/ehost/ pdf? vid $=6$ \&hid $=12 \&$ sid $=8$ edbfce $4-12 b 3-4 c 9 d$ a1b4-8db776d50004\%40sessionmgr102
Steins, G. \& Wicklund, R. A (1998). Motivation in Person Perception: Role of the Other's Perspective. Recuperado el día 20 de abril de 2007 desde: http://web.ebscohost.com/ehost/res ults?vid=3\&hid=103\&sid=6a8914b1-5a82-47c4b0e9-a56be4a7ae95\%40sessionmgr106

Tezer, E. \& Demir, A. (2001). Conflict Behaviors Toward Same-sex and Opposite-Sex Peers Among Male and Female Late Adolescents. Adolescence Fall, 36 (143), 525534. Recuperado el día 17 de enero de 2007 desde: http://web.ebscohost.com/ehost/ pdf? vid=4\&hid=114\&sid=fa6bcca9-5022-4b39b935-26d78b168cdb\%40sessionmgr102

Thornberg, R. (2006). The Situated Nature of Preschool Children's Conflict Strategies. Educational Psychology, 26(1), 109-126.

Troop-Gordon, W. \& Ascher, S. (2005). Modifications in Children's Goals When Encountering Obstacles to Conflict Resolution. Child Development, 76(3), 568-582.

Vernberg, E.M., Ewell, K., Berry, S.H. \& Abwender, D. A. (1994). Sophistication of Adolescent's Interpersonal Negotiation Strategies and Friendships: Formation After Relocation: A Naturally Occurring Experiment. Journal of Research on Adolescent, 4(1), 5-19. 